\title{
COVID-19 disease: novel clinical manifestations and therapeutic exploration
}

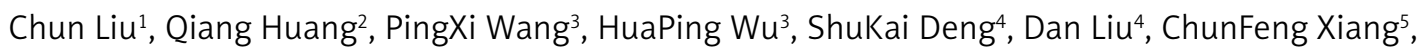
Qiang $\mathrm{Gao}^{1}$, FanWei Zeng ${ }^{3}$, XiangDe Zheng ${ }^{1}$

\author{
${ }^{1}$ Department of Critical Care Medicine, Dazhou Central Hospital, Sichuan Province, China \\ ${ }^{2}$ Department of Critical Care Medicine, Wenjiang District People's Hospital, Chengdu- \\ Sichuan Province, China \\ ${ }^{3}$ Science and Education-Dazhou Central Hospital, Sichuan Province, China \\ ${ }^{4}$ Department of Respiratory Medicine, Affiliated Hospital of Southwest Medical \\ University, Sichuan Province, China \\ ${ }^{5}$ Imaging Center, Dazhou Central Hospital, Sichuan Province, China
}

Submitted: 19 May 2020

Accepted: 3 August 2020

Arch Med Sci

DOI: https://doi.org/10.5114/aoms.2020.98401

Copyright $\odot 2020$ Termedia \& Banach

\section{Abstract}

Introduction: This study aims to provide further clarifications on some new clinical characteristics of COVID-19 recently discovered by our research group.

Material and methods: In this single-centred, retrospective study, we collected all confirmed cases of COVID-19 diagnosed in Dazhou, Sichuan, China from January 23 to February 25, 2020. All the cases were either imported from Wuhan or transmitted in family clusters. We analysed general information on all patients. Meanwhile, the contents of lactic acid, Fib-C, and D-dimer in the serum of patients were detected.

Results: The study included 37 patients diagnosed as having COVID-19, the average age of the patients was $45.76 \pm 13.1$ years. The average positive time of nucleic acid in pharyngeal swabs was $20.65 \pm 6.7$ days. A total of $4(10.8 \%)$ patients were asymptomatic. On serological examination, $33(89.1 \%)$ patients had increased lactic acid, 17 patients had increased fibrinogen $\mathrm{C}$ (Fib-C), and 5 patients had increased D-dimer. Of all patients, 29 were positive for COVID-19-specific antibodies. We also detected COVID-19 nucleic acid in faecal specimens from 4 patients. Klebsiella pneumoniae was found in the sputum of 1 patient. A total of 4 patients received psychological intervention. Lopinavir/ritonavir and Abidol may not be effective in treating COVID-19.

Conclusions: In the serum of most COVID-19-infected patients, lactic acid was higher than normal, and COVID-19-specific antibody was positive. Fib-C and D-dimer in the serum of some infected patients increased. Lopinavir/ ritonavir and Abidol have little effect on shortening the positive time of viral nucleic acid in patients.

Key words: COVID-19, SARS-CoV-2, lactic acid, Fib-C, D-dimer, lopinavir/ ritonavir, Abidol.

\section{Introduction}

In December 2019, a novel severe respiratory syndrome coronavirus 2 (SARS-CoV-2) caused by Coronavirus 2019 (COVID-19) broke out in Wuhan, China. On March 11, 2020, the World Health Organisation de-

\author{
Corresponding authors: \\ FanWei Zeng \\ Science and Education \\ Dazhou Central Hospital \\ Sichuan Province \\ China \\ E-mail:109284521@qq.com \\ XiangDe Zheng \\ Department of \\ Critical Care Medicine \\ Dazhou Central Hospital \\ Sichuan Province, China \\ E-mail:1301889951@qq.com
}


clared COVID-19 a pandemic. Further, as of March 15, 2020, the WHO reported that more than 100 countries had been affected by the COVID-19 pandemic, with about 153,517 confirmed cases and 5735 deaths (https://www.who.int/\#). Previous studies have recognised some clinical characteristics of COVID-19 including the median incubation period estimated to be four days (interquartile range, two to seven) [1], and the most common symptoms are fever, cough, shortness of breath, muscle pain, headache, chest pain, nausea, vomiting, and diarrhoea in some patients. Chest computed tomography (CT) mainly manifests as large patches in both lungs and groundglass opacity. The disease rapidly progresses to acute respiratory distress syndrome (ARDS) and multiple organ failure, which results in death [2]. However, during the treatment process, we discovered some new challenges that need to be addressed.

\section{Material and methods}

\section{Study design and participants}

This is a single-centred, retrospective study. A total of 37 COVID-19 samples were collected

Table I. Demographic characteristics of 37 patients with COVID-19

\begin{tabular}{|c|c|}
\hline General information & $\begin{array}{l}\text { Patients } \\
(n=37)\end{array}$ \\
\hline \multicolumn{2}{|l|}{ Age [years]: } \\
\hline Mean & $45.76 \pm 13.1$ \\
\hline Range & $22-82$ \\
\hline$\leq 39$ & $13(35.1 \%)$ \\
\hline $40-49$ & $11(29.7 \%)$ \\
\hline $50-59$ & $8(21.6 \%)$ \\
\hline $60-69$ & $4(10.8 \%)$ \\
\hline$\geq 70$ & $1(2.7 \%)$ \\
\hline \multicolumn{2}{|l|}{ Sex: } \\
\hline Female & $16(43.2 \%)$ \\
\hline Male & $21(56.8 \%)$ \\
\hline \multicolumn{2}{|l|}{$\begin{array}{l}\text { Pharyngeal swab nucleic acid positive } \\
\text { time: }\end{array}$} \\
\hline Nucleic acid duration range [days] & $6-35$ \\
\hline Average number of days & $20.65 \pm 6.7$ \\
\hline$\leq 15$ days & $10(27 \%)$ \\
\hline$>15$ days & $27(73 \%)$ \\
\hline Number of asymptomatic infections: & $4 / 37(10.8 \%)$ \\
\hline Nucleic acid positive duration & $11-35$ days \\
\hline
\end{tabular}

Nucleic acid positive time calculation method: first nucleic acid positive time to second nucleic acid negative time (diagnosis and treatment of new coronavirus pneumonia in China [trial version 6]). from confirmed patients admitted from January 23 to February 25, 2020, at our hospital, Dazhou Central Hospital, Sichuan Province, China. The hospital admitted a total of 37 COVID-19 patients diagnosed by RT-PCR from the city. The study was approved by the Ethics Committee of Dazhou Central Hospital, and written informed consent was obtained from the patients.

\section{Procedures}

Patient demographics, clinical, laboratory, and other data were collected from the patients' medical records. Their clinical results were followed up until March 18, 2020. All COVID-19 cases were detected from throat swabs, blood, and stool. During hospitalisation, the patients were tested for influenza $A$ virus (H1N1, H3N2), influenza B virus, respiratory syncytial virus, adenovirus, and new coronavirus pneumonia, which are routinely screened in our laboratory. Real-time RT-PCR was used to detect viral nucleic acids from the throat swabs and stool. Also, all the patients underwent a chest CT scan.

\section{Outcomes}

We describe the patient's epidemiological data, abnormal biochemical indicators, the rarely counted pharyngeal swab nucleic acid positive durations, stool nucleic acid positive ratios, and specific antibody test results.

\section{Statistical analysis}

The mean (SD) was used for normally distributed data; otherwise, the median (IQR) and the categorical variable count (\%) were used. Statistical analyses were performed using SPSS (version 19.0) software.

\section{Results}

The study included 37 patients diagnosed with COVID-19, out of which 13 (35.1\%) patients had a clear history of exposure to Wuhan, and the remaining 24 patients were imported second-generation cases. The average age of the patients was $45.76 \pm 13.1$ years, and the maximum age was 82 years. The gender representation of the patients was $56.8 \%$ male and $43.2 \%$ female. The average duration of pharyngeal swabs was $20.65 \pm 6.7$ days. Four (10.8\%) patients were asymptomatic, all females, and the duration of nucleic acid positive in asymptomatic infections was $11-35$ days (Table I).

Elevated admission lactate was reported in 33 (89.1\%) patients, 17 (45.9\%) patients had elevated Fib-C, and 5 (13.5\%) had elevated D-dimers. Based on the new coronavirus-specific antibodies (using the colloidal gold method, Guangzhou Wanfu Biotechnology Co., Ltd., production license 
Table II. Laboratory abnormalities reported among 37 patients with confirmed COVID-19

\begin{tabular}{|c|c|}
\hline Blood biochemistry & Patients \\
\hline \multicolumn{2}{|l|}{ Lactic acid $(0.60-2.20 \mathrm{mmol} / \mathrm{l})$ : } \\
\hline$>$ Normal value & $33 / 37(89.1 \%)$ \\
\hline Mean value & $3.78 \pm 1.26$ \\
\hline \multicolumn{2}{|l|}{ Fib-C (2.03-4.72 g/l): } \\
\hline$>$ Normal value & $17 / 37(45.9 \%)$ \\
\hline Mean value & $4.99 \pm 1.76$ \\
\hline \multicolumn{2}{|l|}{ D-dimer $(0-231 \mathrm{ng} / \mathrm{ml})$ : } \\
\hline$>$ Normal value & $5 / 37(13.5 \%)$ \\
\hline Mean value & $180.36 \pm 166.07$ \\
\hline $\begin{array}{l}\text { Specific antibody positive rate } \\
(\operatorname{lgM}, \lg G)\end{array}$ & $29 / 33(87.9 \%)$ \\
\hline Faeces nucleic acid positive rate: & $4 / 37(10.8 \%)$ \\
\hline Nucleic acid negative time range & $\begin{array}{l}17 \text { days to more } \\
\text { than } 40 \text { days }\end{array}$ \\
\hline
\end{tabular}

number: 20030645), a total of 33 (4 patients did not undergo this test) cases were tested, out of which 29 (87.9\%) tested positive and 4 (10.8\%) were positive for stool nucleic acid (Table II).

During hospitalisation, screening of all the patients for influenza A virus (H1N1, H3N2), influenza $B$ virus, respiratory syncytial virus, and adenovirus antigen/antibody was performed. All the patients tested negative for all the above viruses but showed lung consolidation. Patients with significantly elevated infection parameters had a sputum culture, and patient sputum culture specimens were sent on the $10^{\text {th }}$ day after onset; only $1(2.7 \%)$ of the patients had developed Klebsiella pneumonia with no history of concomitant disease. However, the patient improved after antibiotic treatment.

Among all the COVID-19-confirmed patients, $35(94.6 \%)$ patients were treated with $\alpha$-interferon nebulisation combined with Abidol or lopinavir/ritonavir antiviral therapy. A total of 12 (32.4\%) patients were treated with Abidol and $23(62.2 \%)$ patients with lopinavir/ritonavir. Elevated infection parameters (leukocyte, C-reactive protein, or calcitonin source) or positive sputum culture were reported in 7 (18.9\%) patients. For patients with lower lymphocytes and longer nucleic acid positive duration, human immunoglobulin $10 \mathrm{~g} /$ day was administered for 3-5 days. Only a few patients experienced a transient increase in transaminase and poor appetite during antiviral treatment. Because COVID-19 is a new infectious disease with rapid transmission, psychological intervention is also necessary for some patients who may develop anxiety and depression. A total of $4(10.8 \%)$ patients received psychological inter-
Table III. Co-infection of 37 COVID-19 patients during hospitalisation

\begin{tabular}{|lc|}
\hline Co-infection & Patients \\
\hline Other viruses & 0 \\
\hline Bacteria & Klebsiella pneumonia $(1 / 37,2.7 \%)$ \\
\hline Fungus & 0 \\
\hline
\end{tabular}

Table IV. Treatment strategies for COVID-19 patients during hospitalisation

\begin{tabular}{|lc|}
\hline Treatment & Patients \\
\hline$\alpha$-Interferon nebulisation & $35 / 37(94.6 \%)$ \\
\hline Abidol & $12 / 37(32.4 \%)$ \\
\hline Lopinavir/ritonavir & $23 / 37(62.2 \%)$ \\
\hline Antibiotic & $7 / 37(18.9 \%)$ \\
\hline Human immunoglobulin & $5 / 37(13.5 \%)$ \\
\hline Psychological intervention & $4 / 37(10.8 \%)$ \\
\hline Traditional Chinese medicine & $31 / 37(83.8 \%)$ \\
\hline
\end{tabular}

vention (Table III). Previous studies by Cao et al. have found that lopinavir/ritonavir does not provide good therapeutic benefit in the treatment of patients with severe COVID-19 infection [3]. Our findings were similar to those of previous studies. By comparing the number of nucleic acid-positive days in patients treated with lopinavir/ritonavir, it was found that these drugs had no significant effect on the number of nucleic acid-positive days (Table IV). Previous studies also suggested that Abidol could effectively inhibit COVID-19 replication [4]. However, our study found that its effect on the time of nucleic acid positivity was not significant (Table V).

Typical imaging features from one of the patients with diabetes and SARS-CoV-2 were collected. At onset, the ground-glass opacity of both lungs progressed rapidly and gradually changed. This suggested an increase in exudation in both lungs, reaching a peak of exudation at about 15 days. The entire course of this patient's covid-19 was 2 months (Figure 1).

Table V. Statistics on the use of antiviral drugs in 37 patients with COVID-19

\begin{tabular}{|lcc|}
\hline Drugs & Patients & $\begin{array}{c}\text { Nucleic acid } \\
\text { positive days }\end{array}$ \\
\hline Lopinavir/ritonavir & 23 & $22.29 \pm 7.42$ \\
\hline Unused lopinavir/ritonavir & 14 & $19.65 \pm 6.19$ \\
\hline P-value & & $>0.05$ \\
\hline Abidol & 12 & $22.42 \pm 7.57$ \\
\hline Unused abidol & 25 & $19.80 \pm 6.23$ \\
\hline$P$-value & & $>0.05$ \\
\hline
\end{tabular}





Figure 1. Chest CT. A-D - January 26. E, F - February 6

\section{Discussion}

Coronavirus is an enveloped positive-sense RNA virus, belonging to the Coronaviridae and segment-free virus families [5]. SARS-CoV-2 is among the seven coronaviruses that can infect humans. The other six coronaviruses include HCoV-229E, HCOV-NL63, HCOV-OC43, HCOV-HKU1, SARS-CoV, and MERS-CoV3. SARS-CoV-2, SARS-CoV, and MERS-CoV3 are highly pathogenic and can cause severe human respiratory syndrome, and the other four causes mild upper respiratory disease [610]. The mortality of SARSCOV has been reported to be more than $10 \%$ and MERSCoV at more than $35 \%[11,12]$. However, the SARS-CoV-2 mortality rate is currently estimated at 2-5\% [13].

Previous studies have reported the clinical characteristics and treatment strategies for patients infected with SARS-CoV-2. SARS-CoV-2 can cause coagulopathy and even death [14]. Among COVID-19 patients, 5 (13.5\%) had elevated D-di-

mer, and 17 (45.9\%) had elevated Fib-C. The increase in Fib-C indicated possibilities of blood coagulation; therefore, there is a need to pay close attention to the risk of deep vein thrombosis [15, 16]. Elevated D-dimer is often used as an indicator of pulmonary embolism, but it can also be elevated in inflammatory diseases. Studies by Han et al. $[17,18]$ reported that patients with severe SARS-CoV-2 infection had higher D-dimer. In mild patients, monitoring of the D-dimer values may help in the early detection of severe cases. Gao et al. [19] proposed that D-dimer has important clinical value in early prediction of the severity of COVID-19.

The increase in lactic acid is often caused by anaerobic fermentation. We found that 33 (89.1\%) patients had increased lactic acid, which was mainly accumulated in the lungs. We speculated that in the early stages of the disease, patients experience different levels of hypoxia due to lung infiltration or fever. Therefore, there is a need to 

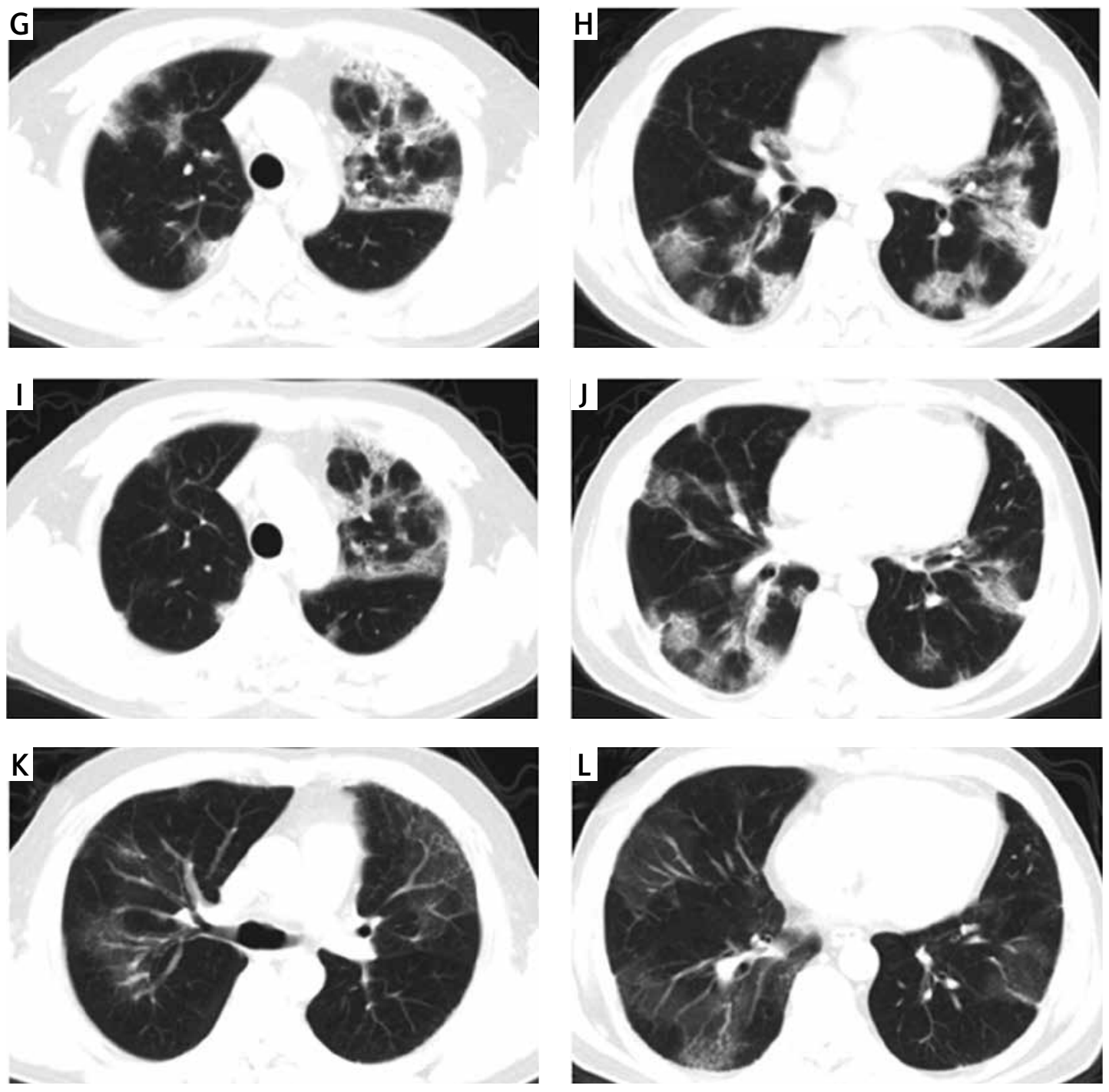

Figure 1. Cont. Chest CT. G, H - February 13, I, J - February 23, K, L - March 18

correct the hypoxia in COVID-19 patients in good time. Peng et al. [20] also found that lactic acid accumulation and thrombosis can exacerbate the risk of death in COVID-19 patients. Therefore, we advocate for close monitoring of the patients' lactic acid, internal environmental conditions, and hypoxia, to prevent the disease from aggravating to multiple organ failure and even death.

Generally, IgM can be produced 3-5 days after exposure to pathogenic microorganisms. IgG usually appears after IgM and can persist for many years after the infection. In this study, 33 patients confirmed to have COVID-19 were tested, and 29 $(87.9 \%)$ were positive or weakly positive. However, Li et al. [21] tested for IgM and IgG antibodies in 25 COVID-19-confirmed patients, and they found $96 \%$ clinical sensitivity and $100 \%$ specificity of the tests. Therefore, detection using the new coronavirus IgM and IgG antibodies can help counteract the inadequacies presented by the nucleic acid detection methods.
Some new challenges currently being faced were identified in this study. For example, among the 37 COVID-19 patients, 4 (10.8\%) had positive nucleic acid in their stool. Zhang et al. [22-24] also reported a small number of cases with positive nucleic acid in stool. One of the patients tested positive for viral nucleic acid in the stool for more than 40 days, and 2 patients experienced intermittent abdominal pain. Therefore, we recommend that the virus in the stool be isolated and cultured from the patient to further confirm the existence of faecal-oral transmission. This will help to provide additional effective measures for the public against the spread of COVID- 19.

Psychological problems caused by COVID-19 in patients cannot be ignored. In this study, we found that 4 (10.8\%) patients experienced severe anxiety and depression and needed psychological intervention to reduce the psychological trauma. At the same time, the patients' infection parameters needed to be closely monitored. COVID-19, 
like other viral types of pneumonia, may present as a viral or bacterial infection at an early stage [2527]. A total of five patients with positive pharyngeal swabs for more than 15 days and a decreased lymph count received intravenous immunoglobulin, and their lymphocyte count was reported to have improved significantly. Fu et al. [28] reported that the use of intravenous immunoglobulin can block FcR activation in the absence of a proven clinical FCR blocker to treat lung inflammation and prevent severe lung injury. Therefore, the intravenous immunoglobulin used in this study may have some effects in preventing disease progression. However, this requires further studies. In addition, previous studies suggested that regulation of renin-angiotensin system (RAS) and lipid might also be a new treatment for COVID-19 [29-31]. By comparing the number of cloudy days of throat swab nucleic acid in patients treated with lopinavir/ritonavir and $\mathrm{Abi}$ dol, it was found lopinavir/ritonavir and Abidol may not be effective in treating COVID-19; however, the number of cases in this study was small, and further research using large sample data should be conducted to determine their effectiveness. In terms of susceptibility to COVID-19, Bianconi et al. found that particulate matter pollution significantly increased the probability of infection with COVID-19 [32]. This may also serve as a warning about the over-modification of nature by human beings. As research on COVID-19 continues to progress [33, 34], we will eventually find treatments for this entirely new virus.

In conclusion, in the serum of most COVID-19 infected patients, lactic acid was higher than normal, and COVID-19-specific antibody was positive. $\mathrm{Fib}-\mathrm{C}$ and D-dimer in the serum of some infected patients increased. Particular attention should be paid to the changes in lactic acid, Fib-C, and D-dimer in patients with COVID-19 to prevent disease progression. The new coronavirus-specific IgM and IgG antibodies can be used to supplement the nucleic acid detection methods for COVID-19 because they provide rapid results. Lopinavir/ritonavir and Abidol have little effect on shortening the positive time of viral nucleic acid in patients.

\section{Acknowledgments}

Chun Liu, Qiang Huang and PingXi Wang contributed equally.

This project was funded by the Science and Technology Bureau of Dazhou City, Sichuan Province, China. However, the funding agency did not play any role in designing the study, data collection and analysis, data interpretation, and report writing.

\section{Conflict of interest}

The authors declare no conflict of interest.

\section{References}

1. Guan WJ, Ni ZY, Hu Y, et al. Clinical characteristics of coronavirus disease 2019 in China. N Engl J Med 2020; 382: $1708-20$

2. Chen N, Zhou M, Dong X, et al. Epidemiological and clinical characteristics of 99 cases of 2019 novel coronavirus pneumonia in Wuhan, China: a descriptive study. Lancet 2020; 395: 507-13.

3. Cao B, Wang Y, Wen D, et al. A trial of lopinavir-ritonavir in adults hospitalized with severe Covid-19. N Engl J Med 2020; 382: 1787-99.

4. Zhu S, Guo X, Geary K, et al. Emerging therapeutic strategies for COVID-19 patients. Discoveries 2020; 8: e105.

5. Zhu N, Zhang D, Wang W, et al. A novel coronavirus from patients with pneumonia in China, 2019. N Engl J Med 2020; 382: 727-33.

6. Liu Z, Xiao X, Wei X, et al. Composition and divergence of coronavirus spike proteins and host ACE2 receptors predict potential intermediate hosts of SARS-CoV-2. J Med Virol 2020; 92: 595-601.

7. Xu J, Zhao S, Teng T, et al. Systematic comparison of two animal-to-human transmitted human coronaviruses: SARS-CoV-2 and SARS-CoV. Viruses 2020; 12: 244.

8. Wang LF, Shi Z, Zhang S, Field H, Daszak P, Eaton BT. Review of bats and SARS. Emerg Infect Dis 2006; 12: 1834-40.

9. Ge XY, Li JL, Yang XL, et al. Isolation and characterization of a bat SARS-like coronavirus that uses the ACE2 receptor. Nature 2013; 503: 535-8.

10. Chen Y, Guo D. Molecular mechanisms of coronavirus RNA capping and methylation. Virol Sin 2016; 31: 3-11.

11. Yin Y, Wunderink RG. MERS, SARS and other coronaviruses as causes of pneumonia. Respirology 2018; 23: 130-7.

12. Song $Z$, Xu Y, Bao L, et al. From SARS to MERS, thrusting coronaviruses into the spotlight. Viruses 2019; 11: 59.

13. Wu YC, Chen CS, Chan YJ. The outbreak of COVID-19: an overview. J Chin Med Assoc 2020; 83: 217-20.

14. New Coronavirus Pneumonia Diagnosis and Treatment Plan (Trial Version 6). Tianjin Journal of Traditional Chinese Medicine: 1-5.

15. Bai $Y$, Shi M, Yang $X$, et al. The value of FDP/FIB and D-dimer/FIB ratios in predicting high-risk APL-related thrombosis. Leuk Res 2019; 79: 34-7.

16. Shi F, Yu A, Yuan L. Clinical significance of detection of coagulation indexes, immune factors and inflammatory factors in patients with pregnancy-induced hypertension syndrome in China. Iran J Public Health 2019; 48: 681-7.

17. Han H, Yang L, Liu R, et al. Prominent changes in blood coagulation of patients with SARS-CoV-2 infection. Clin Chem Lab Med 2020; 58: 1116-20.

18. Zhou F, Yu T, Du R, et al. Clinical course and risk factors for mortality of adult inpatients with COVID-19 in Wuhan, China: a retrospective cohort study. Lancet 2020; 395: 1054-62.

19. Gao Y, Li T, Han M, et al. Diagnostic utility of clinical laboratory data determinations for patients with the severe COVID-19. J Med Virol 2020; 92: 791-6.

20. Peng YD, Meng K, Guan HQ, et al. Clinical characteristics and outcomes of 112 cardiovascular disease patients infected by 2019-nCoV. Zhonghua Xin Xue Guan Bing Za Zhi 2020; 48: E004.

21. Li Q, Liu PB, Qiao ZR, et al. The value of SARS-CoV-2 lgM/ IgG antibody detection in the diagnosis of new coronavirus pneumonia. Int J Labor Med 1-10.

22. Zhang J, Wang S, Xue Y. Fecal specimen diagnosis 2019 novel coronavirus-infected pneumonia. J Med Virol 2020; 92: 680-2. 
23. Xie C, Jiang L, Huang G, et al. Comparison of different samples for 2019 novel coronavirus detection by nucleic acid amplification tests. Int J Infect Dis 2020; 93: 264-7.

24. Zhang W, Du RH, Li B, et al. Molecular and serological investigation of 2019-nCoV infected patients: implication of multiple shedding routes. Emerg Microbes Infect 2020; 9: 386-9.

25. Sarda C, Palma P, Rello J. Severe influenza: overview in critically ill patients. Curr Opin Crit Care 2019; 25: 449-57.

26. LeMessurier KS, Iverson AR, Chang TC, et al. Allergic inflammation alters the lung microbiome and hinders synergistic co-infection with H1N1 influenza virus and Streptococcus pneumoniae in C57BL/6 mice. Sci Rep 2019; 9: 19360.

27. Gu L, Deng H, Ren Z, et al. Dynamic changes in the microbiome and mucosal immune microenvironment of the lower respiratory tract by influenza virus infection. Front Microbiol 2019; 10: 2491.

28. Fu Y, Cheng Y, Wu Y. Understanding SARS-CoV-2-mediated inflammatory responses: from mechanisms to potential therapeutic tools. Virol Sin 2020; 35: 266-71.

29. Cismaru A, Cismaru L, Nabavi SF, et al. Game of "crowning" season 8: RAS and reproductive hormones in COVID-19 - can we end this viral series? Arch Med Sci 2020; DOI: https://doi.org/10.5114/aoms.2020.96604.

30. Katsiki N, Banach M, Mikhailidis DP. Lipid-lowering therapy and renin-angiotensin-aldosterone system inhibitors in the era of the COVID-19 pandemic. Arch Med Sci 2020; 16: 485-9.

31. Reiner Ž, Hatamipour M, Banach M, et al. Statins and the COVID-19 main protease: in silico evidence on direct interaction. Arch Med Sci 2020; 16: 490-6.

32. Bianconi V, Bronzo P, Banach M, et al. Particulate matter pollution and the COVID-19 outbreak: results from Italian regions and provinces. Arch Med Sci 2020; 16: 985-92.

33. Azeez SA, Alhashim ZG, Al Otaibi WM, et al. State-ofthe-art tools to identify druggable protein ligand of SARS-CoV-2. Arch Med Sci 2020; 16: 497-507.

34. Borgio JF, Alsuwat HS, Al Otaibi WM, et al. State-of-theart tools unveil potent drug targets amongst clinically approved drugs to inhibit helicase in SARS-CoV-2. Arch Med Sci 2020; 16: 508-18. 